\title{
Kimura's disease: an unusual cause of cervical tumour
}

\author{
M J Chusid, A L Rock, J R Sty, H W Oechler, D J Beste
}

\begin{abstract}
An 11 year old Chinese boy developed a unilateral cervical mass associated with pronounced eosinophilia and a marked increase in IgE concentrations. A biopsy sample showed massive eosinophilic tissue infiltration consistent with Kimura's disease. This disorder should be suspected when the clinical triad of painless unilateral cervical adenopathy, hypereosinophilia, and hyper-IgE is present, particularly in male Asian patients.

(Arch Dis Child 1997;77:153-154)
\end{abstract}

Keywords: lymphadenopathy; eosinophilia; hyperimmunoglobulinaemia

Kimura's disease is a chronic benign disorder, primarily seen in male Asians during the second and third decades of life. ${ }^{1}$ The major physical manifestation of this disorder is slowly enlarging subcutaneous masses, often in the head and neck area and usually in association with peripheral blood and tissue eosinophilia in combination with markedly increased serum IgE concentrations. ${ }^{1-3}$ Kimura's disease is not often encountered outside the Orient, especially in young children, and thus many paediatricians are unaware of this disease entity. We have treated a young Chinese boy who presented at 8 years of age with an enlarging cervical mass and who was eventually diagnosed as having Kimura's disease.

Children's Hospital of Wisconsin and the Medical College of Wisconsin:

Department of

Pediatrics

M J Chusid

A L Rock

Department of Radiology

J R Sty

Department of

Pathology

$\mathrm{H}$ W Oechler

Department of Otolaryngology D J Beste

Correspondence to: Dr Michael J Chusid, Department of Pediatrics, MFRC, Medical College of Wisconsin, 8701 Watertown Plank Road, Milwaukee, WI 53226, USA.

Accepted 18 April 1997 tive lymphocytes); and platelets, $214 \times 10^{9} / 1$. A rapid mononucleosis assay was negative. Computed tomography of his neck showed promi- nent lymphadenopathy extending from the left lingual space to the thoracic inlet. The largest discrete mass was $2.5 \mathrm{~cm}$ in diameter. No necrosis nor calcification was noted. A chest radiograph was normal.

Because of concern that the mass might be secondary to a neoplasm, a biopsy sample was taken. Microscopic analysis of this material showed the nodal architecture to be intact. Reactive follicular hyperplasia was present with prominent eosinophilia (fig 1). There was no evidence of malignant change. Subsequently, the IgG concentration was seen to be increased at $21.4 \mathrm{~g} / 1$ (normal 5.98-12.05), as were the IgA (3.89 $\mathrm{g} / 1$; normal $0.3-2.0)$ and IgM concentrations (1.52 g/l; normal 46-70). The IgE concentration was markedly increased at $5281 \mu \mathrm{g} / 1$ (normal 30-280). Urine analysis was normal. The following titres were non-reactive: Toxoplasma gondii, rubella, cytomegalovirus, filariasis, Trichinella spiralis, Toxacara canis and catis, and Trypanosoma cruzi.

Based on the peripheral hypereosinophilia, histology, and negative serological testing for parasitic agents, a clinical diagnosis of Kimura's disease was made. For two years the patient's mass stayed approximately the same size. It then began to enlarge painlessly to a size of $8 \times 10 \mathrm{~cm}$. Because of the degree of physical deformity, a decision was made to attempt to resect the mass as completely as possible. Histological examination of the removed tissue showed the same histology as previously noted: maintenance of nodal architecture with the presence of a mixed infiltrate of mature lymphocytes and a large number of eosinophils. Eosinophilic microabscesses were present with central necrosis. Ten months after surgery the boy remains well without any sign of recurrence of the tumour.

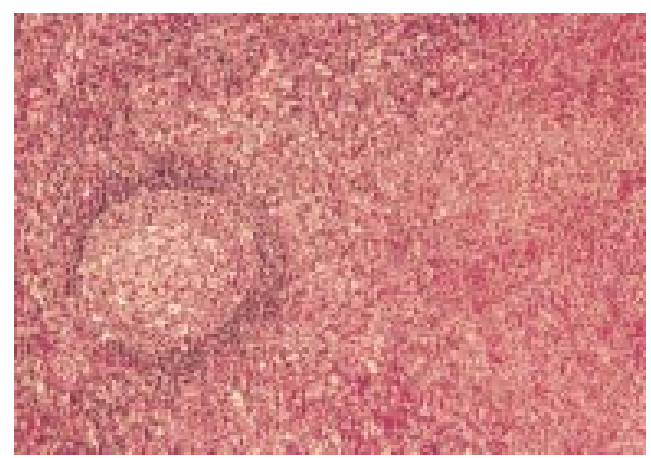

Figure 1 Biopsy sample of cervical mass showing intact follicular architecture with prominent infiltration by eosinophils (haematoxylin and eosin, $\times 100)$. 


\section{Discussion}

Kimura's disease is now recognised as a benign cause of painless localised lymphadenopathy of Asians, especially boys of Chinese and Japanese origin. $^{2-6}$ Although the disease can become apparent at any age, most cases have been reported in the second and third decades of life. The clinical triad of subcutaneous nodules found in the head or neck, prominent peripheral eosinophilia, and highly increased IgE concentrations, particularly when seen in an Asian boy, is highly suggestive of Kimura's disease. Pathologically, nodular lesions usually affect the lymph nodes, though occasionally skin or salivary tissues are affected. ${ }^{23}$ Normal tissue architecture is usually preserved, but follicular hypertrophy of lymphatic tissue with infiltration of lymphocytes, histiocytes, and large numbers of eosinophils is typical. Germinal centres may be necrotic, with central eosinophilic abscesses. ${ }^{23}$

The pathophysiology of Kimura's disease is not understood at this time, but may relate to a disturbance in the normal rate of production of eosinophils and $\operatorname{IgE}$, currently believed to be a product of an interaction between types 1 and 2 (Th1 and Th2) $T$ helper cells. Such a derangement could result in excessive elaboration of eosinophilotrophic cytokines such as interleukin $4 .^{7}$ Patients with Kimura's disease have been shown to have high levels of circulating eosinophilic cationic protein and major basic protein, with heavy concentrations of $\operatorname{IgE}$ in their tissues. ${ }^{2}$ Allergic or parasitic aetiologies for Kimura's disease have been actively sought, but not identified.

The clinical course of Kimura's disease is benign. The subcutaneous masses are usually found in the head and neck region, sometimes affecting the parotid or minor salivary glands. ${ }^{4}$ Infrequently, axillary, inguinal, or epitrochlear nodes may be affected. ${ }^{4}$ Untreated, these masses tend to slowly enlarge and may eventually become disfiguring, as in our patient. Long term, patients seem to do well, although about $10 \%$ may develop a steroid responsive nephrotic syndrome. ${ }^{5}$

Three major therapeutic options exist for Kimura's disease. Resection of the tumour mass may be effective in permanently eradicating the mass if the entire lesion can be removed, but regrowth is common. ${ }^{236}$ Local irradiation has also been shown to be effective in shrinking lesions, but is generally not advocated in younger patients. ${ }^{56}$ Finally, systemic and intralesional corticosteroids have been shown to reduce the size of the lesion, but the tumour tends to recur when these drugs are discontinued. ${ }^{68}$ In selected patients it may be advisable to take a conservative approach, treating only if the mass continues to grow or causes significant deformity.

The differential diagnosis for Kimura's disease includes such entities as eosinophilic granuloma, Mikulicz's disease, acute nonlymphocytic leukaemia, Hodgkin's disease, follicular lymphoma, angioimmunoblastic lymphadenopathy, and angiolymphoid hyperplasia with eosinophilia. Except for angiolymphoid hyperplasia with eosinophilia, the clinical and histological features of these diseases easily distinguishes them from Kimura's disease. Angiolymphoid hyperplasia with eosinophilia is a chronic benign disorder associated with cutaneous nodules heavily infiltrated with eosinophils. It is found in older, mostly female patients, however, and lesions tend to be small and intracutaneous, rather than subcutaneous as in Kimura's disease. ${ }^{9}$ IgE concentrations are usually not increased in angiolymphoid hyperplasia with eosinophilia and there is often no peripheral eosinophilia. ${ }^{9}$

Paediatricians need to be aware of Kimura's disease, especially in view of the growing number of susceptible Asian children present in Western countries. Recognition of this disorder may allow doctors to limit the number of laboratory tests ordered and reduce concern about the possibility of malignant disease. Kimura's disease needs to be strongly considered in any patient, especially an Asian boy, who presents with a painless head or neck mass in association with marked eosinophilia and hyperimmunoglobulinaemia E.

The authors gratefully acknowledge the assistance of Glauco Frizzera MD, Chair, Department of Hematopathology, Armed Forces Institute of Pathology, Washington DC, who reviewed our patient's biopsy specimen.

1 Kimura T, Yoshimura S, Ishikaura E. Unusual granulation combined with hyperplastic change of lymphatic tissue. Trans Soc Pathol Fpn 1948;37:179-80.

2 Motoi M, Horie Y, Akagi T. Kimura's disease: clinical, histological, and immunohistochemical studies. Acta Med Okayama 1992;46:449-55

3 Irish JC, Kain K, Keystone JS, Gullane PJ. Kimura's disease: an unusual cause of head and neck masses. $\mathcal{F}$ Otolaryngol 1994;23:88-91.

4 Som PM, Biller HF. Kimura disease involving parotid gland and cervical nodes: CT and MR findings. $\mathcal{F}$ Comput Assist Tomogr 1992;16:320-2.

5 Atar S, Oberman AS, Ben-Izhak O, Flatou E. Recurrent nephrotic syndrome associated with Kimura's disease in a nephrotic syndrome associated with Kimura's diseas

6 Day TA, Abreo F, Hoajsoe DK, Aarstad RF. Treatment of Kimura's disease: a therapeutic enigma. Otololaryngol Head Neck Surg 1995;112:333-7.

7 Wierenga EA, Backx B, Snoek M, Kapsenberg ML. Relative contributions of human types 1 and 2 T-helper cell-derived eosinophilotrophic cytokines to development of eosinophilia. Blood 1993;82:1471-9.

8 Itami J, Miyoshi T, Ogata H, Miwia K. Radiation therapy in Kimura's disease. Acta Oncol 1989;28:511-4.

9 Ingrams DR, Stafford ND, Creagh TM. Angiolymphoid hyperplasia with eosinophilia. F Laryngol Oncol 1995;109: $262-4$ 